\title{
Application of an online ion-chromatography-based instrument for gradient flux measurements of speciated nitrogen and sulfur
}

Ian C. Rumsey and John T. Walker

Correspondence to: Ian C. Rumsey (rumseyic@cofc.edu)

The copyright of individual parts of the supplement might differ from the CC-BY 3.0 licence. 


\section{Section S2.2 Solutions used in MARGA ion chromatography system}

The MARGA system requires the following chemicals: absorbance solution, internal standard solution, ion chromatography (IC) eluents, and suppressor regenerant. All chemicals are prepared with 18.2 M $\Omega . c m$ double deionized (DDI) water.

The absorbance solution serves as the medium for gases to diffuse into in the WRD and to supersaturate the SJAC to allow particles to grow and thus produce a condensate of water soluble aerosol. It is also used as a carrier liquid to allow the sample to flow through the MARGA system. Absorption solution is made in batches of 20 L using DDI water. Additionally, to prevent the growth of bacteria in the MARGA system, $10 \mathrm{ppm}$ hydrogen peroxide $(0.7 \mathrm{~mL}$ of $30 \% \mathrm{H}_{2} \mathrm{O}_{2}$ ) is added to the absorbance solution. During continuous operation, the MARGA requires $5 \mathrm{~L}$ of absorption solution per day. The absorption solution is replenished ( $35 \mathrm{~L})$ weekly.

As the WRD and SJAC samples are injected from the syringes to the detector box, the sample is automatically mixed with an internal standard that contains a known concentration of lithium bromide ( $\mathrm{LiBr})$. The making of the lithium bromide solution requires two steps. First, a stock solution is prepared by dissolving $4000 \mathrm{mg}$ of $\mathrm{LiBr}(6.94 \% \mathrm{Li}, 79.90 \% \mathrm{Br})$ in a $1 \mathrm{~L}$ volumetric flask, yielding concentrations of $320 \mathrm{mg} \mathrm{L}^{-1} \mathrm{Li}$ and $3680 \mathrm{mg} \mathrm{L}^{-1} \mathrm{Br}$. This stock is stable for at least 12 months, if stored away from light and refrigerated. Internal standard stock solution $(5.6 \mathrm{~mL})$ is then injected into a $5 \mathrm{~L}$ container using a pipette. The container is then filled up with DDI water to a weight of $5600 \mathrm{~g}$. This gives final concentrations of $320 \mu \mathrm{g} \mathrm{L}^{-1}$ for Li and $3680 \mu \mathrm{g} \mathrm{L}^{-1}$ for $\mathrm{Br}$. The $\mathrm{Li}$ and $\mathrm{Br}$ internal standard concentrations were verified by an independent IC system. Stocks are prepared from NIST-traceable LiBr standards (Fisher Scientific, Pittsburgh, PA). The internal standard is replenished bimonthly. 
Separate eluents are needed for both the cation and anion ICs. The cation eluent is

25 prepared by injecting $2.08 \mathrm{~mL}$ of pure methanesulfonic acid (MSA) into a $10 \mathrm{~L}$ container, which

26 is filled with $10 \mathrm{~kg}$ of DDI water. This yields a final MSA concentration of $3.2 \mathrm{mmol} \mathrm{L}^{-1}$. The

27 anion eluent is prepared by adding $8.68 \mathrm{~g}$ of sodium carbonate monohydrate and $6.72 \mathrm{~g}$ of

28 anhydrous sodium bicarbonate to $10 \mathrm{~kg}$ DDI water, which yields a final concentration of 7.0

$29 \mathrm{mmol}$ for sodium carbonate and $8 \mathrm{mmol}$ of sodium bicarbonate. Eluents are prepared from

30

certified chemicals (Fisher Scientific, Pittsburgh, PA). Eluents are replenished biweekly.

To further improve the sensitivity of the anion IC, a suppressor regenerant is used. This

32 chemical improves sensitivity by suppressing eluent background conductivity. An ion-exchange

33 unit is regenerated before each analysis with phosphoric acid. The phosphoric acid in the ion-

34 exchange unit is then rinsed by anion eluent. The suppressor regenerant is made by combining 5

$35 \mathrm{~kg}$ of DDI water and $125 \mathrm{~mL}$ of $85 \%$ phosphoric acid $\left(\mathrm{H}_{3} \mathrm{PO}_{4}\right)$ in a 5-L container, giving a final

36 concentration of $0.35 \mathrm{~mol} \mathrm{~L}^{-1}$ of $\mathrm{H}_{3} \mathrm{PO}_{4}$. The regenerant is made from a certified phosphoric acid

37 stock (Fisher Scientific, Pittsburgh, PA). Regenerant is replenished bimonthly. 


\section{Section S2.3.1 Additional information on external standard liquid solutions}

47 double deionized (DDI) water. The chemicals used were ammonium nitrate $\left(\mathrm{NH}_{4} \mathrm{NO}_{3}\right)($ Fisher

48 Scientific, Pittsburgh, PA), ammonium sulfate $\left((\mathrm{NH} 4)_{2} \mathrm{SO}_{4}\right)$ (Fisher Scientific, Pittsburgh, PA),

49 potassium chloride $(\mathrm{KCl})$ (Fisher Scientific, Pittsburgh, PA), sodium nitrate $\left(\mathrm{NaNO}_{3}\right)($ Fluka, St.

50 Louis, $\mathrm{MO})$, and magnesium sulfate heptahydrate $\left(\mathrm{MgSO}_{4} 7 \mathrm{H}_{2} \mathrm{O}\right)$ (Fluka, St. Louis, $\left.\mathrm{MO}\right)$. All

51 chemicals were ACS (American Chemical Society) grade. Each external standard solution was

52 diluted to the desired concentration using 18.2 M $\Omega . \mathrm{cm}$ double deionized (DDI) water.

53

54

55

56

57

58

59

60

61

62

63

64 


\section{Section S2.3.2 Additional information on methodology used to determine detection limit}

The traditional approach used to determine the detection limit involves combining data from all analytical channels (in this study, there are four different channels including denuder and SJAC samples from both sample boxes) into a single data set. From this single data set, the standard deviation and number of analyses are used to determine the detection limit. The advantage of this approach is that, for an experiment of given duration, a larger number of observation (i.e., degrees of freedom) are available to calculate the detection limit. However, with this approach it is unknown whether the detection limit is strictly a function of the analytical core of the system or is a combination of the analytical and sampling components of the system. In other words, the standard deviation may reflect a combination of random error plus systematic error between channels. To investigate this possibility, the traditional detection limit approach was conducted in conjunction with the Dunn's test (Dunn, 1964) and the Brown-Forsythe test (Brown and Forsythe, 1974) to compare channels. The Dunn's test and the Brown-Forsythe test are non-parametric tests that can be used to determine if there are significant differences in the median concentrations (Dunn's test) and variance (Brown-Forsythe test) across channels. In further detail, the Dunn's test (Dunn, 1964) is a non-parametric statistical test of the difference in medians among multiple groups. It is a rank-sum type test in which the null hypothesis is that the probability of observing a randomly selected value from the one group that is larger than a randomly selected value from another group equals one half. Dunn's test is appropriate for comparing multiple groups consisting of continuous data. In this case, Dunn's test is used to assess whether the differences in analytical detection limits calculated for individual MARGA channels (Denuder1, Denuder2, SJAC1, SJAC2) are at least partially the result of systematic differences (bias) among the channels. The Brown-Forsythe test (Brown and Forsythe, 1974) is a 
88 non-parametric statistical test for the equality of variances among multiple groups. For each

89 observation, a transformation $(\mathrm{z})$ is calculated as its deviation from the corresponding group

90 median. A one-way analysis of variance (ANOVA) is performed on $\mathrm{z}$, in which the F statistic

91 represents the Brown-Forsythe test statistic. In this case, the Brown-Forsythe test is used to

92 assess whether the differences in analytical detection limits calculated for individual MARGA

93 channels (Denuder1, Denuder2, SJAC1, SJAC2) are at least partially the result of differences in

94 precision (variance) among the channels.

95

96

97

98

99

100

101

102

103

104

105

106

107 
123

124

125

126

127

128

129

130

131

132

133

134

135

136

137

138

139

140

29

31

32

35

36

38

39

40

\section{Section S3.1.1 Tables and figures associated with the accuracy results}

Table S1. MARGA blank concentrations expressed as equivalent air concentration for each sample box (SB).

\begin{tabular}{|c|c|c|c|c|c|c|c|c|}
\hline \multicolumn{9}{|c|}{ Blank concentration $\left(\mu \mathrm{g} \mathrm{m}^{-3}\right)$} \\
\hline & $\mathrm{NO}_{3}^{-}$ & $\mathrm{HNO}_{3}$ & $\mathrm{SO}_{4}^{2-}$ & $\mathrm{SO}_{2}$ & $\mathrm{NH}_{4}^{+}$ & $\mathrm{NH}_{3}$ & $\mathrm{Na}^{+}$ & $\mathrm{K}^{+}$ \\
\hline $\begin{array}{l}\text { SB1 } \\
\text { SB }\end{array}$ & $0.001^{\mathrm{a}}$ & 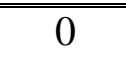 & 0.040 & 0.037 & 0 & 0 & 0 & 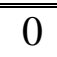 \\
\hline & $0.006^{\mathrm{b}}$ & 0.003 & 0.013 & 0.012 & 0 & 0 & 0 & 0 \\
\hline & $33^{\mathrm{c}}$ & 37 & 33 & 37 & 33 & 37 & 33 & 37 \\
\hline SB2 & 0.001 & 0 & 0.034 & 0.035 & 0 & 0.001 & 0 & 0 \\
\hline & 0.003 & 0.001 & 0.015 & 0.011 & 0 & 0.005 & 0 & 0 \\
\hline & 37 & 35 & 37 & 35 & 37 & 35 & 37 & 35 \\
\hline
\end{tabular}

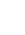

5

6

28

0

33

37 
141 Table $\mathrm{S}_{2} . \mathrm{HNO}_{3}$ and $\mathrm{NO}_{3}{ }^{-}$external standard runs and the difference between expected and 142 observed concentration.

\begin{tabular}{ccccccc}
\hline & \multicolumn{2}{c}{ SB1 concentrations $\left(\mu \mathrm{g} \mathrm{m}^{-3}\right)$} & \multicolumn{2}{c}{ SB2 concentrations $\left(\mu \mathrm{g} \mathrm{m}^{-3}\right)$} \\
Exp $^{\mathrm{a}}$ & Obs $^{\mathrm{b}}$ & $\begin{array}{c}\text { Difference } \\
\text { (offset) }\end{array}$ & Exp & Obs & $\begin{array}{c}\text { Difference } \\
\text { (offset) }\end{array}$ \\
\hline \multirow{6}{*}{$\mathrm{HNO}_{3}$} & 0 & 0 & 0 & 0 & 0 & 0 \\
& 0.133 & 0.192 & 0.060 & 0.128 & 0.181 & 0.053 \\
& 0.380 & 0.528 & 0.149 & 0.366 & 0.502 & 0.136 \\
& 1.345 & 1.523 & 0.178 & 1.303 & 1.479 & 0.176 \\
$\mathrm{NO}_{3}{ }^{-}$ & 2.302 & 2.483 & 0.181 & 2.219 & 2.404 & 0.186 \\
& 0 & 0.001 & 0.001 & 0 & 0.001 & 0.001 \\
& 0.131 & 0.186 & 0.05 & 0.126 & 0.183 & 0.057 \\
& 1.324 & 0.518 & 0.144 & 0.360 & 0.488 & 0.128 \\
& 2.266 & 2.487 & 0.163 & 1.283 & 1.435 & 0.153 \\
\hline
\end{tabular}

$143 \quad{ }^{a}$ Expected concentration.

144 b Observed concentration.

$145{ }^{\mathrm{c}}$ Difference between expected and observed concentration (i.e. observed concentration minus expected 146 concentration).

147

148

149

150 


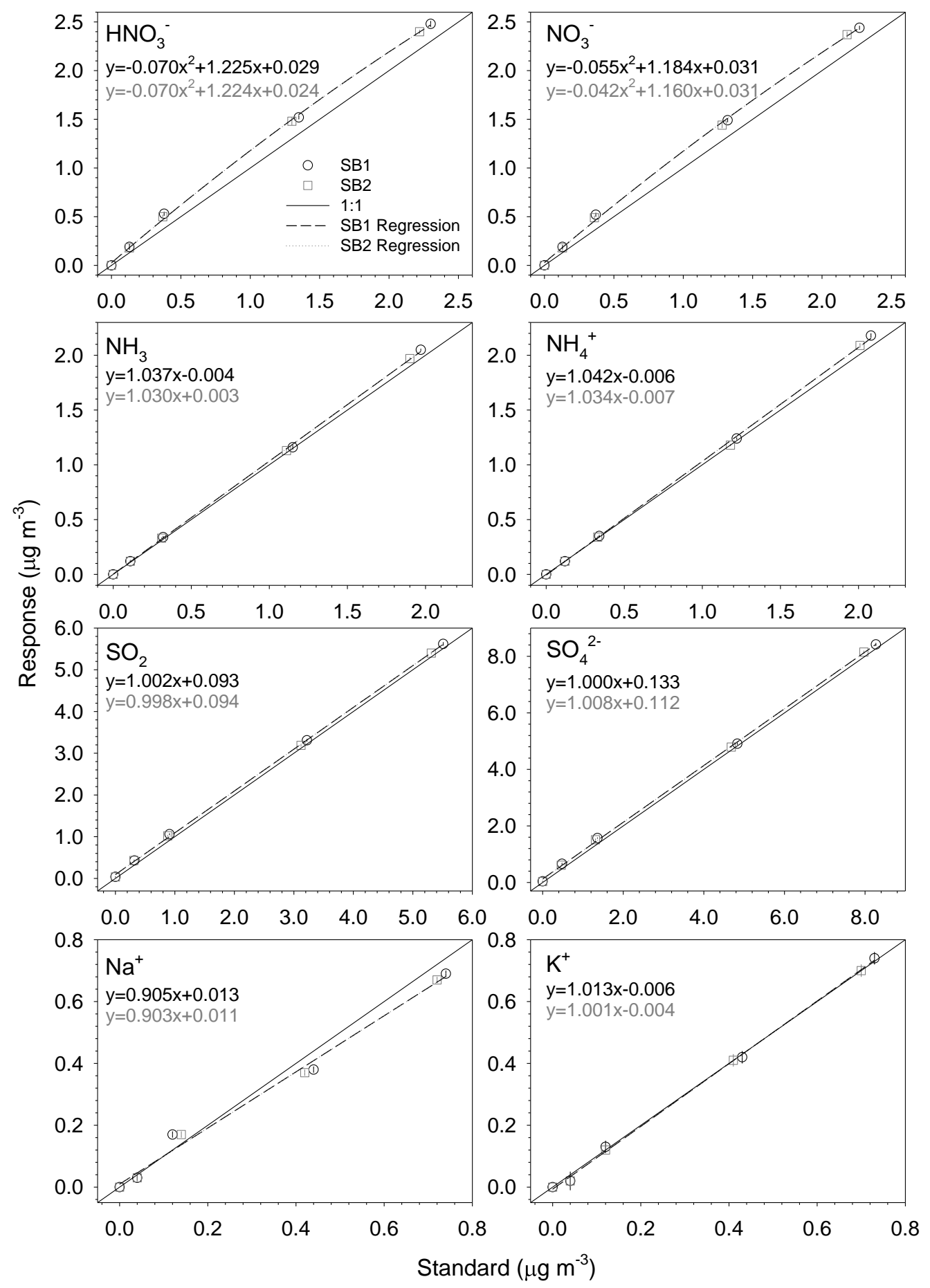

176 Figure S1. Results of external standard tests. Response is given in equivalent air concentration $177\left(\mu \mathrm{g} \mathrm{m}^{-3}\right)$.

178 

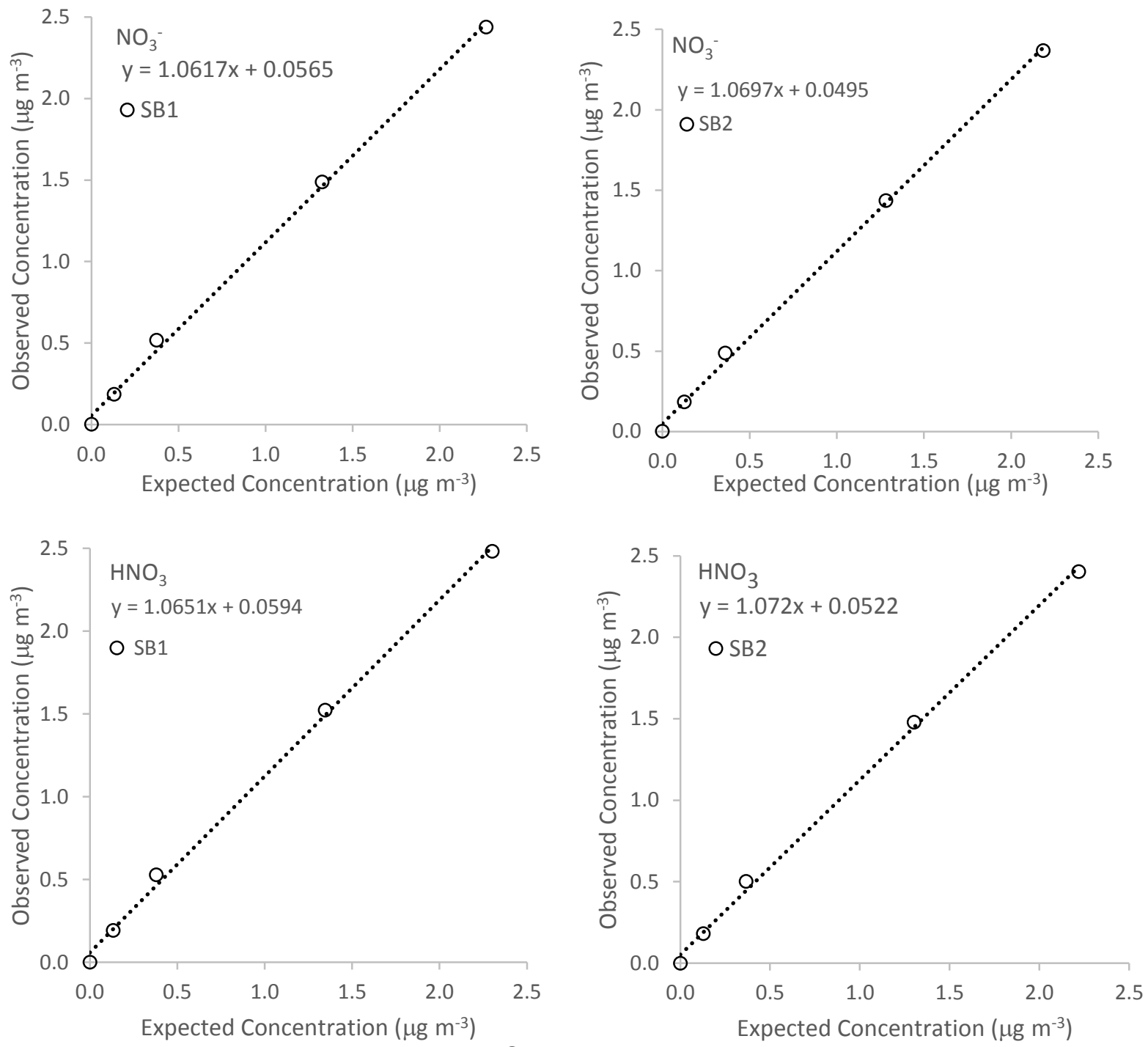

182

183 Figure $\mathrm{S} 2 . \mathrm{NO}_{3}{ }^{-}$linear regression analysis for external standard tests. 
The results of the Dunn's test and Brown-Forsythe test for each analyte are presented in Table S3.

Table S3. Results of the Dunn's test (DU) and Brown-Forsythe test (BF) for individual MARGA channels. Top row indicates channels being compared: S1 = SJAC Sample Box 1, D1 = Denuder Sample Box 1, etc. For the Brown-Forsythe test, p values are reported with bold indicating statistical significance at the $10 \%$ level. For Dunn's test, N and D indicated not significantly different versus significantly different, respectively.

\begin{tabular}{cccccccc}
\hline & & $\mathrm{S} 1-\mathrm{S} 2$ & $\mathrm{~S} 1-\mathrm{D} 1$ & $\mathrm{~S} 1-\mathrm{D} 2$ & $\mathrm{~S} 2-\mathrm{D} 1$ & $\mathrm{~S} 2-\mathrm{D} 2$ & $\mathrm{D} 1-\mathrm{D} 2$ \\
\hline \hline $\mathrm{NO}_{3}{ }^{-}$ & $\mathrm{DU}$ & $\mathrm{N}$ & $\mathrm{N}$ & $\mathrm{N}$ & $\mathrm{N}$ & $\mathrm{N}$ & $\mathrm{N}$ \\
& $\mathrm{BF}$ & $\mathbf{0 . 0 9}$ & 0.86 & 0.45 & $\mathbf{0 . 0 8}$ & $\mathbf{0 . 0 4}$ & 0.60 \\
$\mathrm{SO}_{4}{ }^{2-\mathrm{a}}$ & $\mathrm{DU}$ & $\mathrm{N}$ & $\mathbf{D}$ & $\mathbf{D}$ & $\mathbf{D}$ & $\mathbf{D}$ & $\mathrm{N}$ \\
& $\mathrm{BF}$ & 0.80 & 0.18 & 0.24 & 0.29 & 0.38 & 0.88 \\
$\mathrm{SO}_{4}{ }^{2-}$ & $\mathrm{DU}$ & $\mathrm{N}$ & $\mathbf{D}$ & $\mathbf{D}$ & $\mathbf{D}$ & $\mathbf{D}$ & $\mathrm{N}$ \\
& $\mathrm{BF}$ & 0.80 & 0.18 & 0.24 & 0.29 & 0.38 & 0.88 \\
$\mathrm{NH}_{4}{ }^{+}$ & $\mathrm{DU}$ & $\mathrm{N}$ & $\mathrm{N}$ & $\mathbf{D}$ & $\mathrm{N}$ & $\mathbf{D}$ & $\mathbf{D}$ \\
& $\mathrm{BF}$ & 0.71 & $\mathbf{0 . 0 4}$ & $\mathbf{0 . 0 1}$ & $\mathbf{0 . 1 0}$ & $\mathbf{0 . 0 2}$ & 0.35 \\
$\mathrm{Na}^{+}$ & $\mathrm{DU}$ & $\mathrm{N}$ & $\mathbf{D}$ & $\mathbf{D}$ & $\mathbf{D}$ & $\mathrm{N}$ & $\mathrm{N}$ \\
& $\mathrm{BF}$ & 0.86 & 0.48 & 0.84 & 0.65 & 0.97 & 0.70 \\
$\mathrm{Na}^{+}$ & $\mathrm{DU}$ & $\mathrm{N}$ & $\mathrm{N}$ & $\mathrm{N}$ & $\mathrm{N}$ & $\mathrm{N}$ & $\mathrm{N}$ \\
& $\mathrm{BF}$ & 0.28 & 0.15 & 0.22 & $\mathbf{0 . 0 2}$ & $\mathbf{0 . 0 5}$ & 0.89 \\
$\mathrm{~K}^{+}$ & $\mathrm{DU}$ & $\mathrm{N}$ & $\mathrm{N}$ & $\mathrm{N}$ & $\mathrm{N}$ & $\mathrm{N}$ & $\mathrm{N}$ \\
& $\mathrm{BF}$ & 0.19 & 0.85 & 0.72 & 0.26 & 0.35 & 0.87 \\
\hline
\end{tabular}

${ }^{a}$ Detection limits for $\mathrm{SO}_{4}{ }^{2-}$ and $\mathrm{Na}^{+}$were determined using two liquid standards with different concentrations.

The results of Dunn's test and Brown-Forsythe test (Table S3) indicate that the sampling components of the MARGA are influencing the detection limit of all the compounds except $\mathrm{K}^{+}$.

The influence of the sampling components of the MARGA on the detection limit varies from compound to compound. In both $\mathrm{SO}_{4}{ }^{2-}$ detection limit analyses there were multiple channels that had significant differences in median concentrations. For $\mathrm{NO}_{3}{ }^{-}$, the detection limit was not influenced by differences in channel medians, but was influenced by differences in the channel variance with three of the six channel to channel comparisons having a Brown-Forsythe p-value less than 0.10. It is not known what is causing the significant differences in channel variance, but 
212 it is hypothesized that inconsistency in peak integration may be an issue. Differences in channel 213 median concentrations and/or concentration variance also influenced the determined detection 214 limit for $\mathrm{Na}^{+}$and $\mathrm{NH}_{4}{ }^{+}$. It can therefore be concluded that analytical detection limits calculated 215 by combining channels is function of variability related to random error in individual channels, 216 systematic differences across channels, and differences in variance across channel 


\section{Section S3.1.2.b Detection limits for individual MARGA channels}

218 Table S4. Detection limit results for individual MARGA channels.

\begin{tabular}{|c|c|c|c|c|c|c|c|c|}
\hline & & $\begin{array}{c}\text { Exp conc }^{\mathrm{b}} \\
\left(\mu \mathrm{L} \mathrm{L}^{-1}\right)\end{array}$ & $\begin{array}{c}\text { Median Obs } \\
\left(\mu \mathrm{g} \mathrm{L}^{-1}\right)\end{array}$ & $\begin{array}{l}\begin{array}{l}S t D^{d} \\
\left(\mu \mathrm{g} \mathrm{L}^{-1}\right)\end{array} \\
\end{array}$ & $\mathrm{N}^{\mathrm{e}}$ & T-stat & $\begin{array}{c}\text { Liq Conc DL } \\
\quad\left(\mu \mathrm{g} \mathrm{L}^{-1}\right)\end{array}$ & $\begin{array}{c}\text { Air Conc DL } \\
\left(\mu \mathrm{g} \mathrm{m}^{-3}\right)\end{array}$ \\
\hline & $\overline{\mathrm{D} 1}$ & & 7.64 & 0.76 & 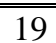 & 21.33 & 2.02 & 0.050 \\
\hline \multirow[t]{4}{*}{$\mathrm{NO}_{3}^{-}$} & D2 & 5.34 & 8.05 & 0.65 & 14 & 1.35 & 1.76 & 0.044 \\
\hline & S1 & & 7.60 & 0.75 & 19 & 1.33 & 2.00 & 0.050 \\
\hline & S2 & & 7.89 & 1.14 & 20 & 1.33 & 3.03 & 0.076 \\
\hline & Average $\mathrm{NO}_{3}{ }^{-} / \mathrm{HNO}_{3}$ & & & & & & 2.20 & $0.055 / 0.056$ \\
\hline & D1 & & 2.15 & 0.74 & 37 & 1.31 & 1.93 & 0.048 \\
\hline \multirow[t]{5}{*}{$\mathrm{SO}_{4}^{2-\mathrm{a}}$} & D2 & 0.00 & 2.05 & 0.70 & 35 & 1.31 & 1.83 & 0.046 \\
\hline & S1 & & 1.65 & 0.54 & 33 & 1.31 & 1.41 & 0.035 \\
\hline & S2 & & 1.49 & 0.62 & 37 & 1.31 & 1.62 & 0.040 \\
\hline & Average $\mathrm{SO}_{4}^{2-} / \mathrm{SO}_{2}$ & & & & & & 1.70 & $0.042 / 0.028$ \\
\hline & D1 & & 26.40 & 0.63 & 19 & 1.33 & 1.68 & 0.042 \\
\hline \multirow[t]{5}{*}{$\mathrm{SO}_{4}^{2-}$} & D2 & 19.47 & 27.06 & 0.98 & 14 & 1.35 & 2.65 & 0.066 \\
\hline & S1 & & 27.39 & 1.08 & 19 & 1.33 & 2.87 & 0.072 \\
\hline & S2 & & 25.92 & 0.63 & 20 & 1.33 & 1.67 & 0.042 \\
\hline & Average $\mathrm{SO}_{4}{ }^{2--} \mathrm{SO}_{2}$ & & & & & & 2.21 & $\mathbf{0 . 0 5 5 / 0 . 0 3 7}$ \\
\hline & D1 & & 5.11 & 0.26 & 19 & 1.33 & 0.69 & 0.017 \\
\hline \multirow[t]{5}{*}{$\mathrm{NH}_{4}^{+}$} & D2 & 4.91 & 5.51 & 0.33 & 14 & 1.35 & 0.89 & 0.022 \\
\hline & S1 & & 4.93 & 0.17 & 20 & 1.33 & 0.45 & 0.011 \\
\hline & S2 & & 4.91 & 0.19 & 20 & 1.33 & 0.50 & 0.013 \\
\hline & Average $\mathrm{NH}_{4}+/ \mathrm{NH}_{3}$ & & & & & & 0.63 & $0.016 / 0.015$ \\
\hline & D1 & & 1.63 & 0.49 & 19 & 1.33 & 1.30 & 0.033 \\
\hline \multirow{5}{*}{$\mathrm{Na}^{+}$} & D2 & 1.75 & 1.53 & 0.45 & 14 & 1.35 & 1.22 & 0.030 \\
\hline & S1 & & 1.39 & 0.35 & 20 & 1.33 & 0.93 & 0.023 \\
\hline & S2 & & 1.42 & 0.45 & 20 & 1.33 & 1.19 & 0.030 \\
\hline & Average & & & & & & 1.16 & 0.029 \\
\hline & D1 & & 7.25 & 0.28 & 20 & 1.33 & 0.74 & 0.019 \\
\hline \multirow[t]{5}{*}{$\mathrm{Na}^{+}$} & D2 & 5.00 & 7.16 & 0.20 & 21 & 1.33 & 0.53 & 0.013 \\
\hline & S1 & & 7.03 & 0.44 & 18 & 1.33 & 1.17 & 0.029 \\
\hline & S2 & & 7.01 & 0.54 & 21 & 1.33 & 1.43 & 0.036 \\
\hline & Average & & & & & & 0.97 & 0.024 \\
\hline & D1 & & 5.14 & 0.64 & 20 & 1.33 & 1.70 & 0.042 \\
\hline \multirow[t]{4}{*}{$\mathrm{K}^{+}$} & D2 & 4.91 & 5.68 & 0.58 & 21 & 1.33 & 1.54 & 0.038 \\
\hline & S1 & & 5.03 & 0.67 & 18 & 1.33 & 1.79 & 0.045 \\
\hline & S2 & & 5.31 & 0.53 & 21 & 1.33 & 1.40 & 0.033 \\
\hline & Average & & & & & & 1.61 & 0.040 \\
\hline
\end{tabular}


227 Section S3.1.2.c Additional analysis of the detection limits in comparison to previous

228 studies

229 Thomas et al. (2009) and Wolff et al. (2010) determined detection limits in field

230 conditions using the GRAEGOR system, which is quasi-similar to the MARGA system.

231 However, they used different methodologies to determine their detection limit. Therefore, in

232 order to make an equivalent comparison, the detection limits were also calculated for this study

233 using the Thomas et al. (2009) and Wolff et al. (2010) detection limit methodologies. Thomas et

234 al. (2009) calculated average blanks using average blank values plus three standard deviations of

235 the blank value, whereas Wolff et al. (2010) used three standard deviations of the blank value.

236 The results are shown in Table S5.

Table S5. Calculate detection limits from this study using different methodologies

\begin{tabular}{|c|c|c|c|c|c|c|c|}
\hline & \multicolumn{3}{|c|}{$\begin{array}{l}\text { Detection limit calculation } \\
\text { for data in this study using } \\
\text { different methodologies ( } \mu \mathrm{g} \\
\left.\qquad \mathrm{m}^{-3}\right)\end{array}$} & \multicolumn{4}{|c|}{$\begin{array}{l}\text { Previous studies detection limits } \\
\qquad\left(\mu \mathrm{g} \mathrm{m}^{-3}\right)\end{array}$} \\
\hline & \multirow[t]{2}{*}{$\begin{array}{l}\text { This } \\
\text { study }\end{array}$} & \multirow{2}{*}{$\begin{array}{c}\text { Thomas } \\
\text { et al. } \\
\text { (2009) }\end{array}$} & \multirow{2}{*}{$\begin{array}{l}\text { Wolff } \\
\text { et al. } \\
(2010)\end{array}$} & \multicolumn{2}{|c|}{$\begin{array}{l}\text { Thomas et } \\
\text { al. (2009) }\end{array}$} & \multicolumn{2}{|c|}{$\begin{array}{l}\text { Wolff et } \\
\text { al. }(2010)\end{array}$} \\
\hline & & & & MPIC & $\mathrm{CEH}$ & NEU & EGER \\
\hline $\mathrm{NO}_{3}^{-}$ & 0.056 & 0.066 & 0.065 & "0.077 & 0.150 & 0.093 & 0.130 \\
\hline $\mathrm{HNO}_{3}$ & 0.057 & 0.066 & 0.066 & 0.079 & 0.191 & 0.094 & 0.132 \\
\hline $\mathrm{SO}_{4}{ }^{2-\mathrm{a}}$ & 0.048 & 0.093 & 0.056 & 0.201 & 0.116 & - & - \\
\hline $\mathrm{SO}_{2}$ & 0.032 & 0.073 & 0.037 & 0.123 & 0.254 & - & - \\
\hline $\mathrm{SO}_{4}{ }^{2-}$ & 0.064 & 0.112 & 0.075 & - & - & - & - \\
\hline $\mathrm{SO}_{2}$ & 0.043 & 0.086 & 0.050 & - & - & - & - \\
\hline $\mathrm{NH}_{4}{ }^{+}$ & 0.021 & 0.025 & 0.025 & 0.117 & 0.180 & 0.074 & 0.022 \\
\hline $\mathrm{NH}_{3}$ & 0.020 & 0.024 & 0.023 & 0.111 & 0.161 & 0.055 & 0.021 \\
\hline $\mathrm{Na}^{+}$ & 0.029 & 0.030 & 0.030 & - & - & - & - \\
\hline $\mathrm{Na}^{+}$ & 0.026 & 0.033 & 0.033 & - & - & - & - \\
\hline $\mathrm{K}^{+}$ & 0.038 & 0.038 & 0.045 & - & - & - & - \\
\hline
\end{tabular}


240 In summary, regardless of the detection limit methodology, the detection limits determined in

241 this study are lower than those determined by Thomas et al. (2009) and Wolff et al. (2010) for all

242 nitrogen and sulfur compounds with the exception of $\mathrm{NH}_{3} . \mathrm{NH}_{3}$ detection limits determined in

243 this study are lower than those reported by Thomas et al. (2009) and Wolff et al. (2010) at the

244 grassland NEU site, but similar to those reported by Wolff et al. (2010) at the forest (EGER) site.

245

246

247

248

249

250

251

252

253

254

255

256

257

258

259

260

261

262

263

264

265

266 
267 Section S3.2.1 Tables and figures associated with the concentration gradient precision

268 (gradient detection limit) results

269

270 Table S6. Orthogonal least squares regression coefficients comparing SB1 (x) to SB2 (y).

271 Results are reported for individual colocation experiments during June-July (Period 1), August

272 (Period 2), and October, 2012 (Period 3). Number of hourly observations for each period are 273 approximately 87,138 , and 73 , respectively.

\begin{tabular}{lcccccc}
\hline Period & 1 & 2 & 3 & 1 & 2 & 3 \\
\hline & ------- & Slope & ------- & ------ & $\begin{array}{c}\text { Intercept } \\
\left(\mu \mathrm{g} \mathrm{m}^{-3}\right)\end{array}$ & ------- \\
\hline $\mathrm{NH}_{3}$ & 0.94 & 1.03 & 0.95 & -0.10 & -0.01 & -0.08 \\
$\mathrm{NH}_{4}{ }^{+}$ & 1.06 & 1.01 & 1.02 & -0.03 & -0.01 & -0.01 \\
$\mathrm{HNO}_{3}$ & 0.86 & 1.02 & 1.00 & 0.05 & -0.02 & -0.01 \\
$\mathrm{NO}_{3}{ }^{-}$ & 0.87 & 1.06 & 1.03 & 0.02 & -0.02 & 0.01 \\
$\mathrm{SO}_{2}$ & 0.97 & 1.04 & 1.03 & -0.02 & -0.01 & -0.05 \\
$\mathrm{SO}_{4}{ }^{2-}$ & 1.02 & 0.99 & 1.01 & 0.09 & 0.05 & 0.01 \\
\hline
\end{tabular}

274

275

276

277

278

279

280

281

282

283

284

285

286

287

288 

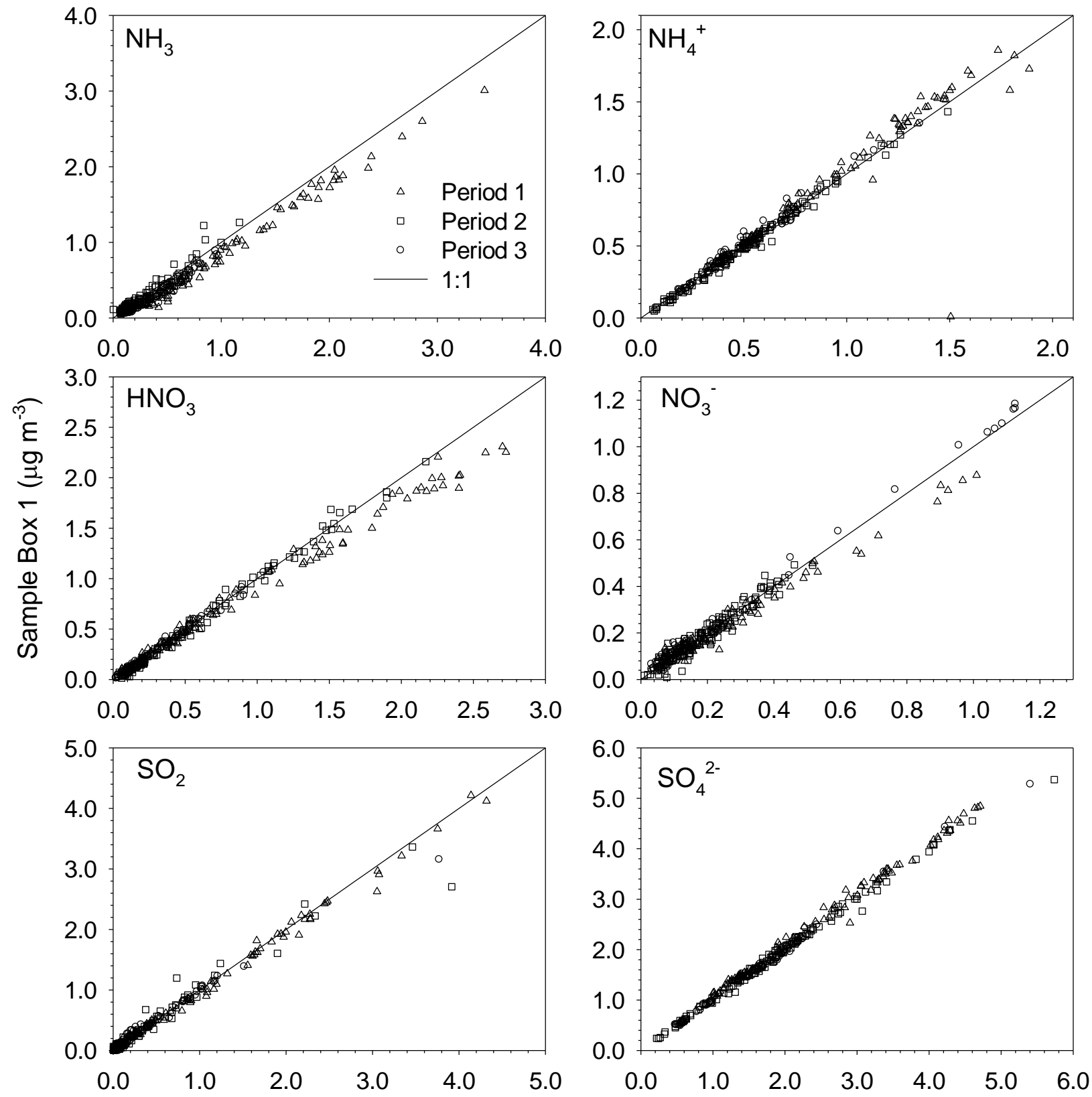
Sample Box $2\left(\mu \mathrm{g} \mathrm{m}^{-3}\right)$

291 Figure S3. Scatterplots of concentrations measured by MARGA sample boxes 1 and 2 during 292 three colocation experiments represented by different symbols (Period 1= June-July 2012; Period 2932 =August 2012; Period 3= October 2012) 

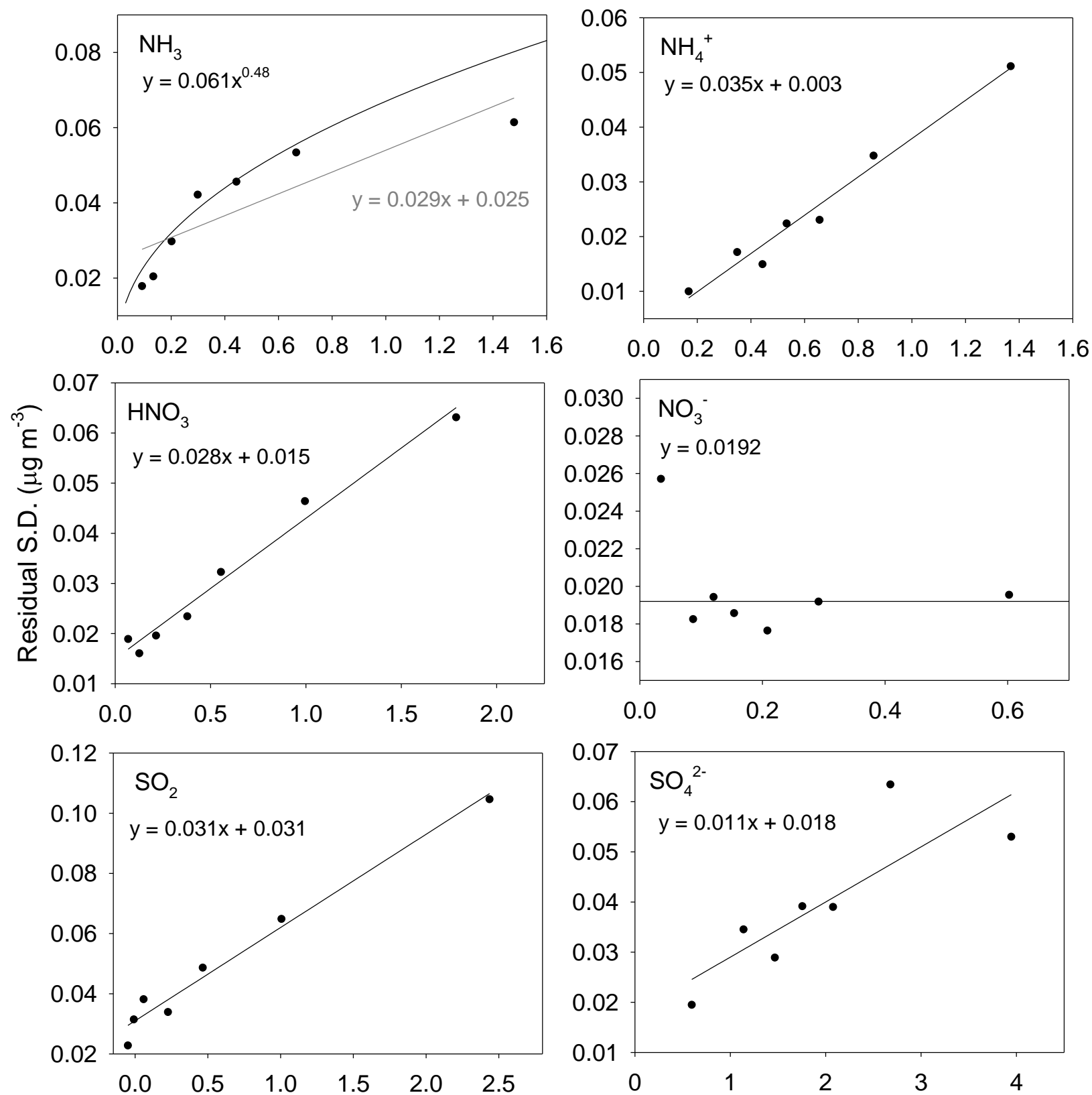

Concentration $\left(\mu \mathrm{g} \mathrm{m}^{-3}\right)$

Figure S4. Curves describing relationship between air concentration and the standard deviation deviation of residual and corresponding average air concentration. Bins comprise $\approx 42$ observations. 


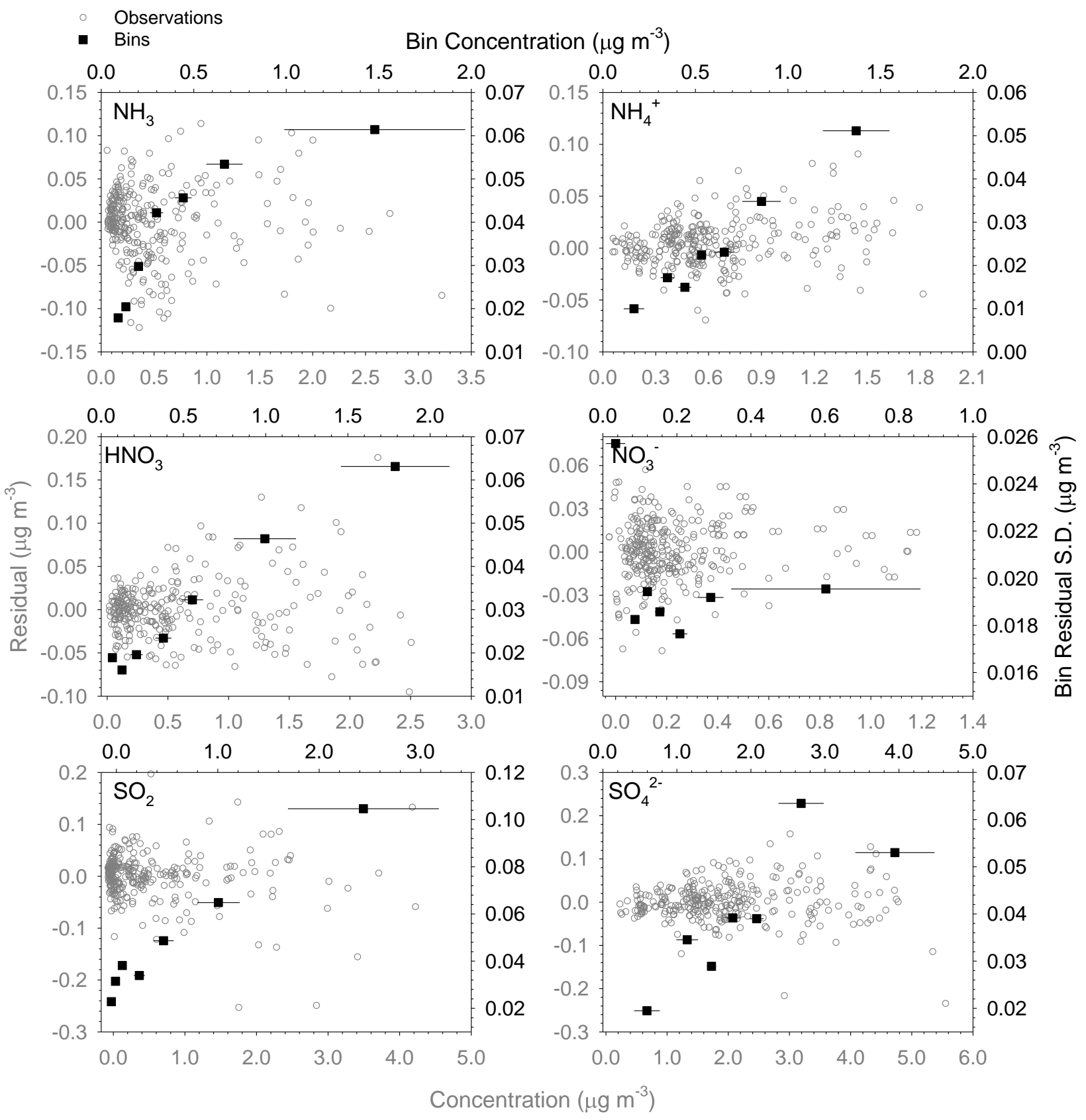

305

306

307

308

309

310

311

312

Figure S5. Scatterplots (gray circles) of orthogonal least squares residuals versus average concentration between sample boxes. Data from three colocation experiments are combined. Black squares represent binwise standard deviation of residuals versus concentration. Horizontal bars represent standard deviation of concentration within bin. Individual bins contain $\approx 42$ observations. 


\section{Section S3.3.2.a Tables and figures associated with flux uncertainty results}

314 Table S7. Summary statistics for select meteorological variables, air concentrations, and fluxes during the period 23 September - 14

315 October, 2012.

\begin{tabular}{|c|c|c|c|c|c|c|c|c|c|c|c|c|c|c|c|}
\hline & \multicolumn{3}{|c|}{ Meteorology } & \multicolumn{6}{|c|}{$\begin{array}{l}\text { Concentration } \\
\qquad\left(\mu \mathrm{g} \mathrm{m}^{-3}\right)\end{array}$} & \multicolumn{6}{|c|}{$\begin{array}{c}\text { Flux } \\
\left(\mathrm{ng} \mathrm{m}^{-2} \mathrm{~s}^{-1}\right)\end{array}$} \\
\hline & $\begin{array}{l}\text { W.S. } \\
\left(\mathrm{m} \mathrm{s}^{-1}\right)\end{array}$ & $\begin{array}{c}\mathrm{u} * \\
\left(\mathrm{~m} \mathrm{~s}^{-1}\right)\end{array}$ & $\begin{array}{c}\text { Air Temp. } \\
\left({ }^{\circ} \mathrm{C}\right)\end{array}$ & $\mathrm{NH}_{3}$ & $\mathrm{NH}_{4}{ }^{+}$ & $\mathrm{HNO}_{3}$ & $\mathrm{NO}_{3}^{-}$ & $\mathrm{SO}_{2}$ & $\mathrm{SO}_{4}{ }^{2-}$ & $\mathrm{NH}_{3}$ & $\mathrm{NH}_{4}^{+}$ & $\mathrm{HNO}_{3}$ & $\mathrm{NO}_{3}^{-}$ & $\mathrm{SO}_{2}$ & $\mathrm{SO}_{4}{ }^{2-}$ \\
\hline Mean & 0.8 & 0.1 & 15.9 & 0.4 & 0.7 & 0.3 & 0.4 & 0.5 & 1.9 & 9.1 & -1.0 & -4.3 & -3.1 & -5.5 & -4.0 \\
\hline Median & 0.7 & 0.1 & 15.5 & 0.3 & 0.7 & 0.2 & 0.3 & 0.1 & 1.8 & 3.9 & -0.4 & -1.8 & -1.6 & -1.9 & -2.4 \\
\hline S.D. ${ }^{\mathrm{a}}$ & 0.5 & 0.1 & 5.8 & 0.3 & 0.3 & 0.3 & 0.3 & 1.1 & 0.8 & 11.7 & 2.1 & 6.4 & 4.3 & 16.8 & 5.1 \\
\hline Max. & 2.4 & 0.5 & 28.7 & 1.6 & 1.5 & 1.8 & 1.2 & 8.8 & 4.3 & 82.8 & 6.6 & 6.6 & 18.1 & 46.7 & 17.7 \\
\hline Min. & 0.0 & 0.0 & 4.4 & 0.0 & 0.2 & 0.0 & 0.0 & 0.0 & 0.5 & -1.4 & -14.6 & -38 & -35.4 & -245 & -28.9 \\
\hline $\mathrm{N}^{\mathrm{b}}$ & 474 & 474 & 474 & 449 & 376 & 451 & 384 & 451 & 384 & 406 & 358 & 407 & 366 & 407 & 366 \\
\hline $\mathrm{D} .=$ & ada & & & & & & & & & & & & & & \\
\hline W.S. $=$ & Speed & & & & & & & & & & & & & & \\
\hline
\end{tabular}



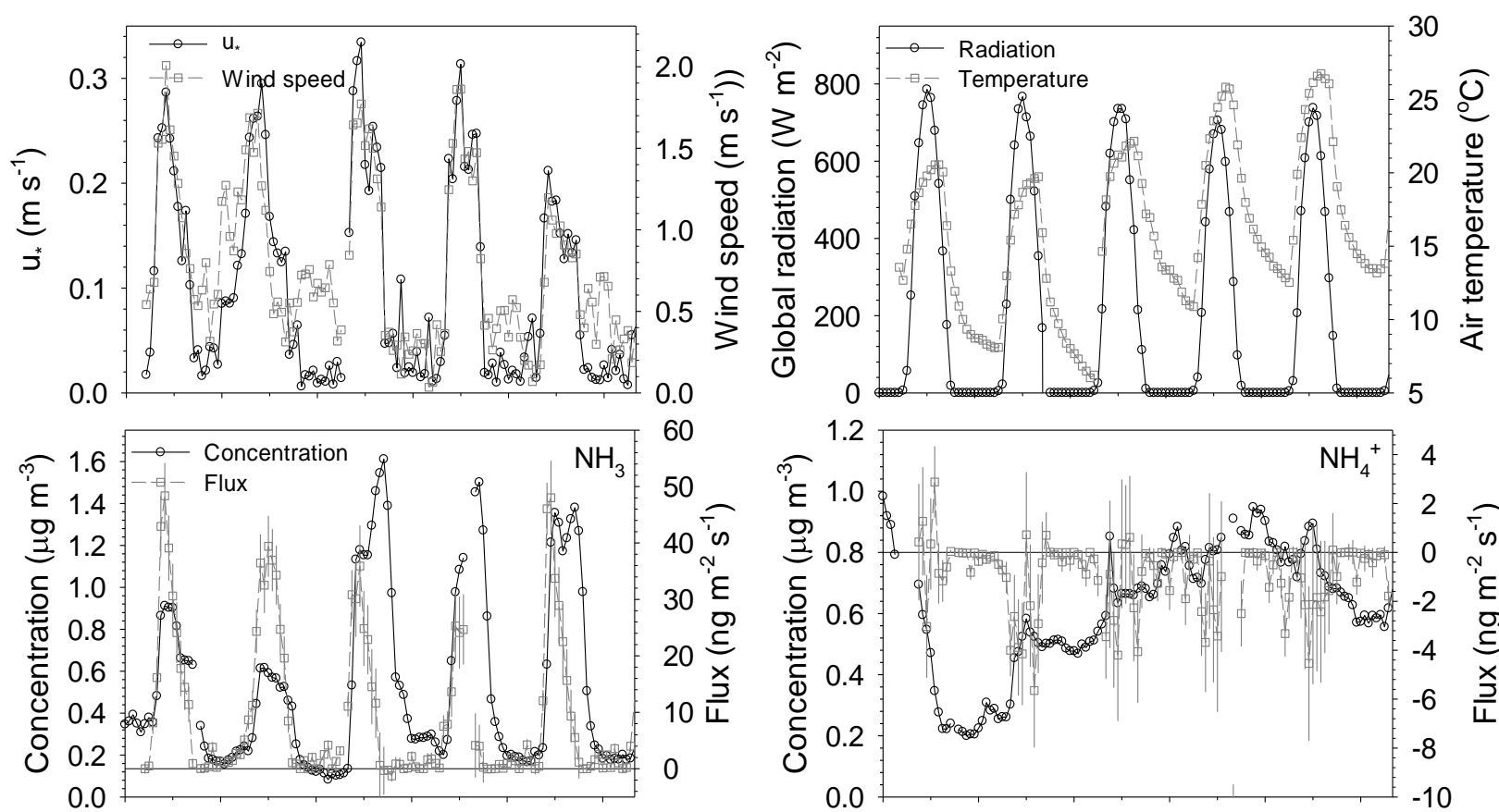

60
50
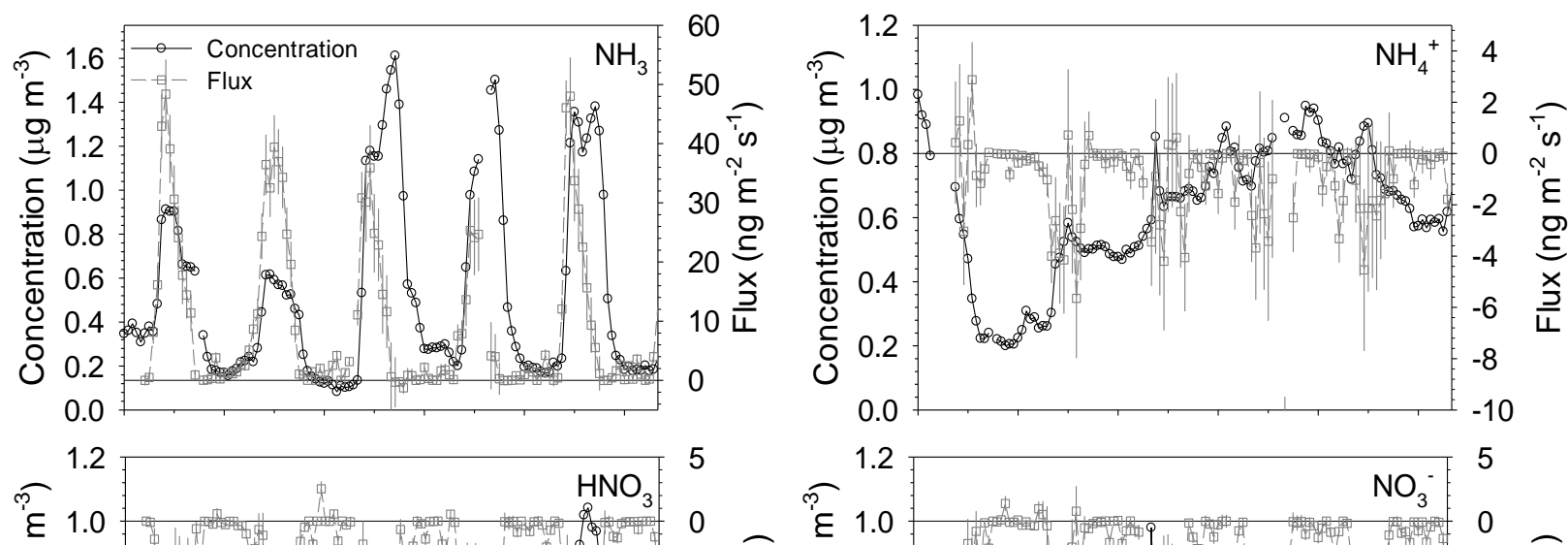

5
$0 \simeq$
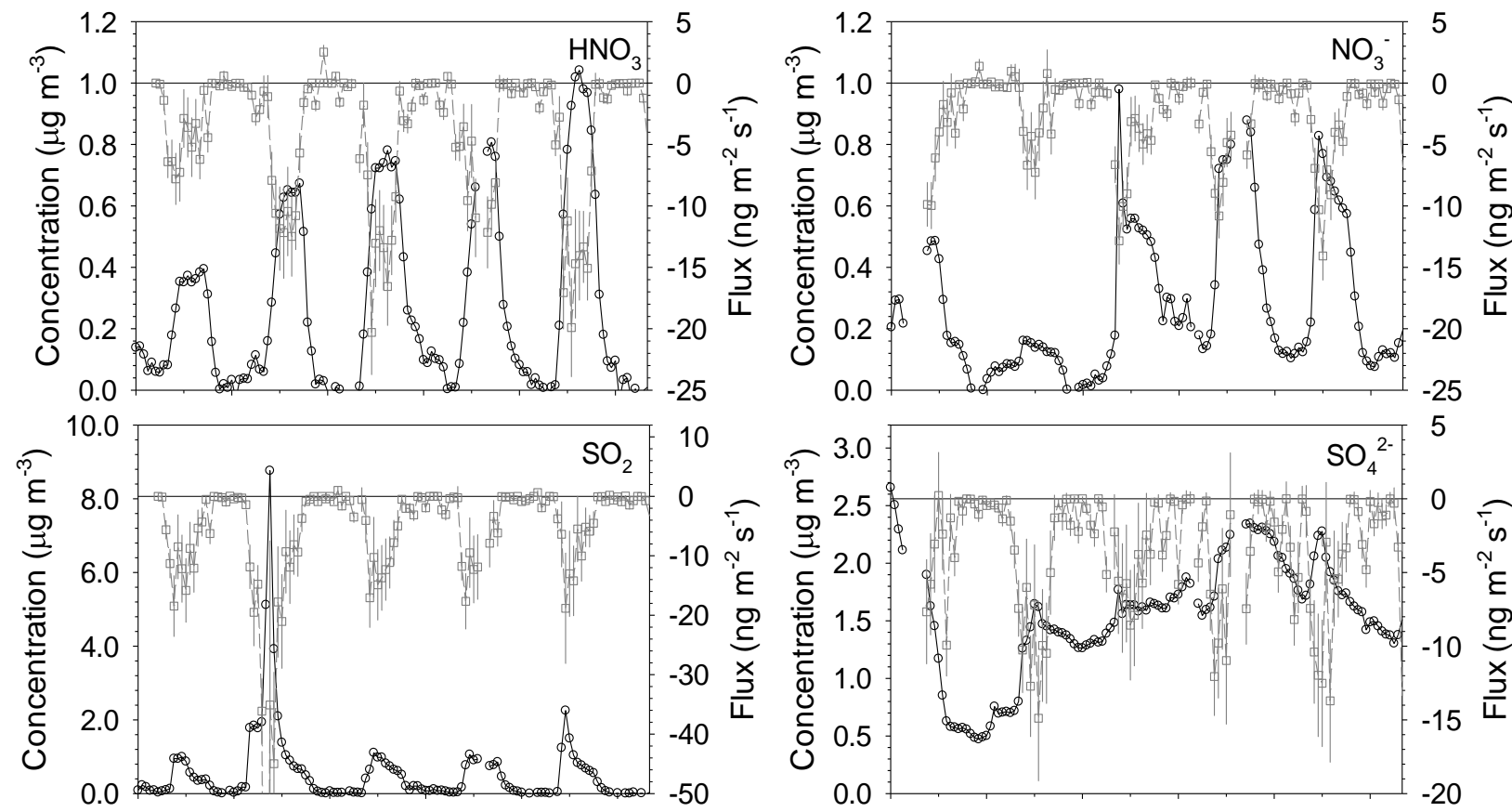

$$
10
$$
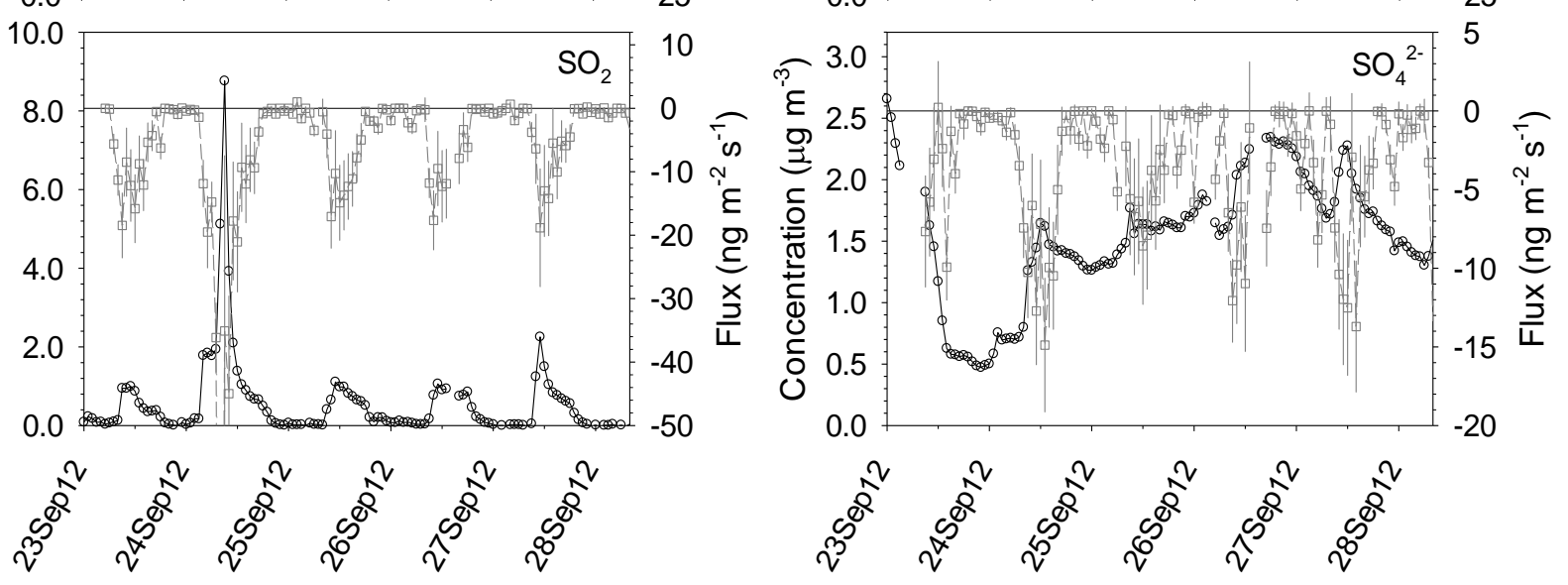

320 Figure S6. Example time series of meteorological variables, air concentrations, and fluxes.

321 Fluxes also include flux error calculated from equation (4) in the main text. For plots of

322 chemical species, concentrations and fluxes are represented by black circles and gray squares,

323 respectively. 
324 Section S3.2.2.b Analysis of the influence of the co-location concentration adjustment on 325 calculated fluxes

The influence of the co-location concentration adjustment on calculated fluxes was

327 determined by performing a sensitivity analysis in which hypothetical colocation corrections 328 were applied to the three weeks of flux data presented in the manuscript. For this analysis, a 329 reasonable range of slope and intercept (offset) values were determined based on co-location 330 slope and offset values from this manuscript (see supplemental Table S6). The influence of co331 location slope and offset values on overall average fluxes was quantified as the percent change in 332 the average flux, with test values of slope and offset applied, relative to the average flux with a 333 slope and offset of 1 and 0, respectively. Results are presented in figure S7.

Results indicate that the correction for a co-location slope of 1.1 (assuming an offset of 335 0.0) can change the average flux by $30 \%$ for $\mathrm{HNO}_{3}$ and approximately a factor of 4.5 for $\mathrm{NH}_{4}{ }^{+}$.

336 Correcting for an offset of 0.1 (assuming a slope of 1.0), can change the average flux by $70 \%$ for $337 \mathrm{SO}_{2}$ and approximately a factor of 7 for $\mathrm{NH}_{4}{ }^{+}$. Because concentration gradients are small relative 338 to the air concentration, the colocation correction can be large, particularly for aerosols, which 339 deposit more slowly than gases thereby resulting in smaller concentration gradients. It should be 340 noted that, because concentrations and fluxes (i.e., concentration gradients) vary by site, the 341 importance of the colocation test will also vary by site. However, the sensitivity analysis 342 demonstrates that colocation tests are needed to help control the accuracy of the concentration gradient measurement. 

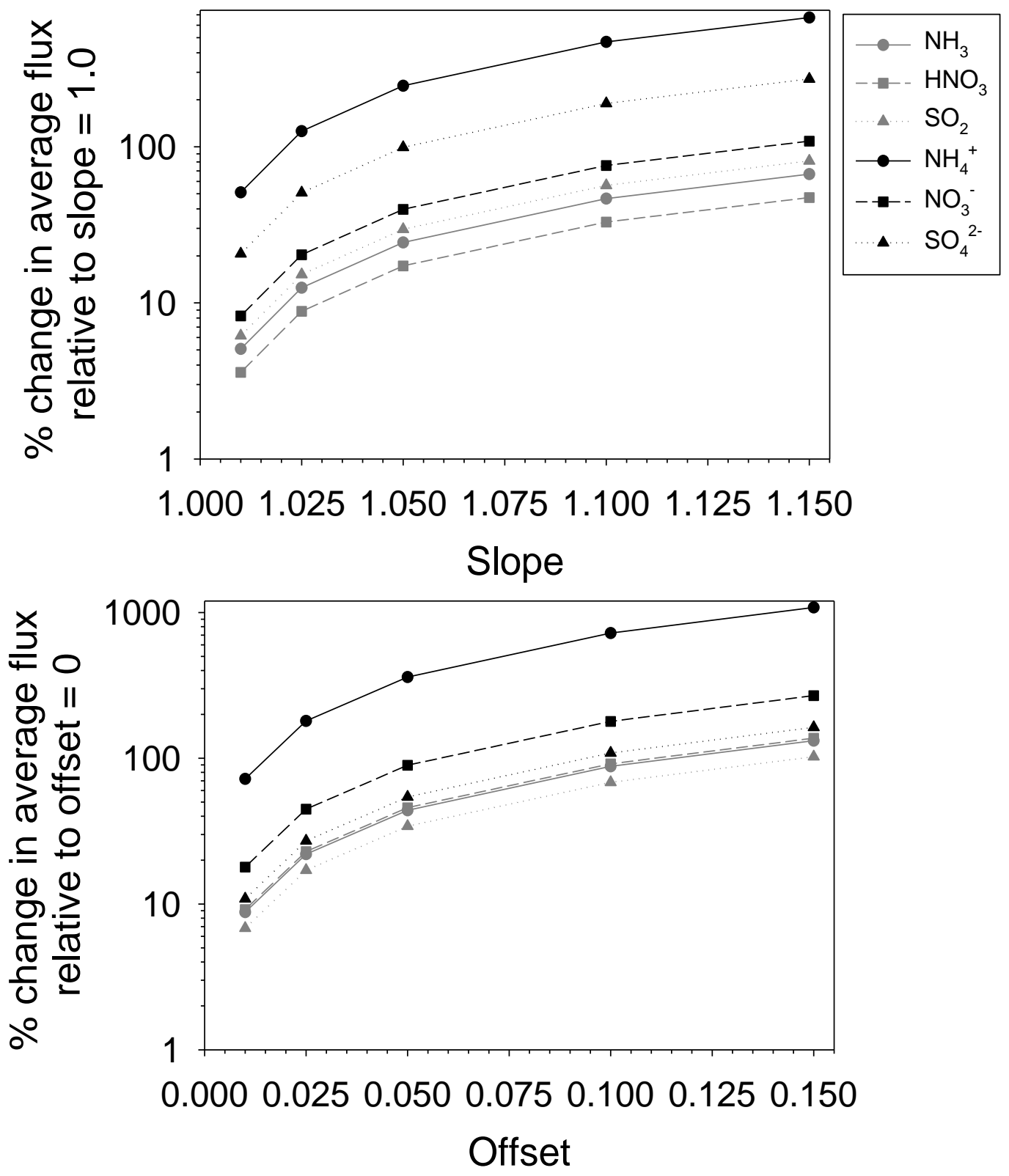

352 Figure S7: Results of co-location sensitivity analysis in which hypothetical values of slope and 353 offset (intercept) were applied to the observed fluxes. 


\section{Supporting Information References}

359 Brown, M.B., Forsythe, A.B. Robust tests for equality of variances. J. Am. Statist. Assoc. 69 (346), 364-367, DOI 10.1080/01621459.1974.10482955, 1974.

361

362

363

364

365

366

367

368

369

370

371

372

373

Dunn, O.J. Multiple comparisons using rank sums. Technometrics 6 (3), 241-252, DOI 10.1080/00401706.1964.10490181, 1964.

Thomas, R.M., Trebs, I., Otjes, R., Jongejan, P.A.C., ten Brink, H., Phillips, G., Kortner, M., Meixner, F.X., Nemitz, E. An automated analyzer to measure surface-atmosphere exchange fluxes of water soluble inorganic aerosol compounds and reactive trace gases. Environ. Sci. Technol., 43 (5), 1412-1418, DOI 10.1021/es8019403, 2009.

Wolff, V., Trebs, I., Ammann, C., Meixner, F.X. Aerodynamic gradient measurements of the $\mathrm{NH}_{3}-\mathrm{HNO}_{3}-\mathrm{NH}_{4} \mathrm{NO}_{3}$ triad using a wet chemical instrument: an analysis of precision requirements and flux errors. Atmos. Meas. Tech., 3 (1), 187-208, DOI 10.5194/amt-3-187$2010,2010$. 